(C) 1998 International Press

Adv. Theor. Math. Phys. 2 (1998) 559-569

\title{
Quark-Monopole Potentials in Large $N$ Super Yang-Mills
}

\author{
Joseph A. Minahan \\ Physics Department \\ U.S.C., University Park \\ Los Angeles, CA 90089-0484, U. S. A. \\ and \\ Institute for Theoretical Physics, UCSB \\ Santa Barbara, CA 93106
}

\begin{abstract}
We compute the quark-monopole potential for $\mathcal{N}=4$ super YangMills in the large $N$ limit. We find an attractive potential that falls off as $1 / L$ and is manifestly invariant under $g \rightarrow 1 / g$. The strength of the potential is less than the quark-antiquark and monopole-antimonopole potentials.
\end{abstract}

\section{Introduction}

There has been a flurry of activity in large $N$ conformal Yang-Mills theories [1-24]. This was started by Maldacena's remarkable observation [2] that computations in strongly coupled large $N$ Yang-Mills with $N=4$ supersymmetry can be mapped onto tree level computations in type IIB supergravity living on $A d S^{5} \times S^{5}$. The radii of the 5-sphere and anti-de Sitter space are equal and are given by $\left(4 \pi g^{2} N\right)^{1 / 4}=\left(g_{Y M}^{2} N\right)^{1 / 4}$. Hence, if $g_{Y M}^{2} N>>1$ then the corresponding supergravity theory is weakly coupled and so tree

e-print archive: http://xxx.lanl.gov/abs/hep-th/9803111 
level supergravity computations should give accurate results for strongly coupled Yang-Mills.

This identification of $N=4$ Yang-Mills with IIB Supergravity has led to nontrivial predictions for large $N$ Yang-Mills. These are the first predictions in strongly coupled Yang-Mills that go beyond the level of the BPS states.

In particular, Rey and Yee [13] and Maldacena [14]were able to compute the coulomb potential between a very massive quark and antiquark at strong coupling. The basic idea was to compute the Nambu-Goto action in an anti-de Sitter background for a static string configuration. The result is an energy that diverges, but after subtraction of the quark masses, one is left with a finite attractive potential that falls off as $1 / L$, where $L$ is the distance between the quarks on the D3-branes.

In this paper we will extend the result of [13] and [14] to the case of a massive quark and monopole. We consider a brane configuration consiting of $N$ D3 branes at the origin and another D3 brane taken out to infinity. This describes an $\mathcal{N}=4 U(N) \times U(1)$ gauge theory. The BPS spectrum contains heavy quarks and monopoles that transform under the fundamental of $U(N)$ and are charged under the $U(1)$ gauge symmetry. The relevant string configuration is a $\mathbf{Y}$ junction [25-34], where one string coming out of the junction is attached to one of the $N$ D3 branes at the origin. The other two strings are attached to the D3 brane at infinity, but at a finite distance $L$ from each other along the brane ${ }^{1}$. After subtraction of the monopole and quark mass, we are left with a finite attractive potential between the particles. We find an explicit function for the attractive potential as a function of the coupling which is manifestly $S$ dual under $g \rightarrow 1 / g$.

That the potential is attractive is not surprising. A quark with mass $\phi$ can bind with a monopole of mass $\phi / g$ to form a dyon with mass $\phi \sqrt{1+1 / g^{2}}$. We should also expect that the attractive potential between a quark and a monopole is not as strong as the potential between a quark and an antiquark, since the latter two objects can annihilate completely. We will show that this potential is indeeed less than the $Q \bar{Q}$ potential.

In section 2 we review the calculation in $[13,14]$. In section 3 we extend this to the problem of a quark-monopole pair.

\section{Quark-Antiquark Potential}

Let us review the calculation in $[13,14]$. We will follow closely the argument of [14]. The metric for type IIB string theory in the presence of $N$ D3-branes was computed by Horowitz and Strominger. As we move down the throat,

\footnotetext{
${ }^{1}$ Rey and Yee considered using a junction configuration to describe baryons [13]
} 
we can approximate the metric by that for $A d S^{5} \times S^{5}$ with radii $R$. For the Nambu-Goto computation we will use the Euclidean version:

$$
d s^{2}=\alpha^{\prime}\left[\frac{U^{2}}{R^{2}}\left(d t^{2}+d x_{i} d x_{i}\right)+R^{2} \frac{d U^{2}}{U^{2}}+R^{2} d \Omega_{5}^{2}\right]
$$

where $R=(4 \pi g N)^{1 / 4}$ and $g$ is the string coupling. The world-sheet action for the string is

$$
S=\frac{1}{2 \pi \alpha^{\prime}} \int d \tau d \sigma \sqrt{\operatorname{det}\left[G_{M N} \partial_{\alpha} X^{M} \partial_{\beta} X^{N}\right]}
$$

where $G_{M N}$ is the metric in (2.1). To find a static configuration we set $\tau=t$ and $\sigma=x$, where $x$ is a direction along the D3-branes. We assume that one of the D3-branes has been taken out to $U=\infty$ and that the string configuration starts and ends on this brane. Then the action simplifies to

$$
S=\frac{1}{2 \pi} \int d t d x \sqrt{\left(\partial_{x} U\right)^{2}+U^{4} / R^{4}}
$$

Since the configuration is static, the integration over $t$ leads to a constant T. $U(x)$ is minimized as a function of $x$ if

$$
\frac{U^{4}}{\sqrt{\left(\partial_{x} U\right)^{2}+U^{4} / R^{4}}}=U_{0}^{2} / R^{2}
$$

where $U_{0}$ is a constant to be determined. Hence we can write $x$ as a function of $U$

$$
x=\frac{R^{2}}{U_{0}} \int_{1}^{U / U_{0}} \frac{d y}{y^{2} \sqrt{y^{4}-1}} .
$$

We can then find $U_{0}$ by setting $x$ to $x=L / 2$ which corresponds to moving half way along the string. The middle of the string is then at $U=U_{0}$. Hence we find that

$$
L=\frac{2 R^{2}}{U_{0}} \int_{1}^{\infty} \frac{d y}{y^{2} \sqrt{y^{4}-1}}=\frac{R^{2}}{U_{0}} \frac{(2 \pi)^{3 / 2}}{\Gamma(1 / 4)^{2}} .
$$

The total energy is found by substituting (2.5) with the condition (2.6) into (2.3). The resulting energy is infinite, because the masses of the quarks have been included in the total energy. To find the quark mass, consider a string configuration that runs from a D3 brane at large but finite $U=U_{\text {max }}$, to the $N \mathrm{D} 3$ branes at $U=0$, and with a fixed coordinate $x_{i}$ in the directions along the branes. Hence we can replace $\sigma$ by $U$ in (2.2) and we find that the energy of this configuration is $U_{\max } / 2 \pi$. This is the quark mass. Subtracting 
this off from the total energy and letting $U_{\max } \rightarrow \infty$, we find that the remaining energy is

$$
\begin{array}{r}
E_{Q \bar{Q}}=\frac{2}{2 \pi} \int_{U_{0}}^{\infty} d U\left(\frac{U^{2} / U_{0}{ }^{2}}{\sqrt{U^{4} / U_{0}^{4}-1}}-1\right)-U_{0} \\
=-U_{0} \frac{\sqrt{2 \pi}}{(\Gamma(1 / 4))^{2}}=-\frac{4 \pi^{2}\left(g_{Y M}^{2} N\right)^{1 / 2}}{L(\Gamma(1 / 4))^{4}} .
\end{array}
$$

The result in (2.7) is consistent with $S$ duality. To see this consider the situation where the quarks are replaced with monopoles. We then want to find a minimum energy configuration for this system. Now instead of a fundamental string, we should consider a D-string attached to the brane out at infinity. The world sheet action is exactly the same as in (2.2), except for an extra factor of $1 / g=4 \pi / g_{Y M}^{2}$. Hence the coulomb energy for the monopole-monopole pair is

$$
E_{M \bar{M}}=-\frac{16 \pi^{3}\left(g_{Y M}^{-2} N\right)^{1 / 2}}{L(\Gamma(1 / 4))^{4}}
$$

Hence, under the $S$ dual transformation $g \rightarrow 1 / g$ the potential in (2.7) is transformed into the potential in (2.8).

Note that the results in (2.7) and (2.8) are both valid if $g N>>1$ and $N / g>>1$. This is certainly true if $N$ is large and $g \sim 1$.

\section{The Quark-Monopole Potential}

In this section we derive the potential for a heavy quark and monopole.

As in the previous section, we have $N$ D3 branes at $U=0$ and one D3 brane at $U=U_{\max } \rightarrow \infty$. We assume that there is a heavy quark at $x=0$ and a heavy monopole at $x=L$. Both the quark and monopole transform under the fundamental representation of $S U(N)$. The string configuration looks as follows: In the $(x, U)$ plane we have a fundamental string (i.e. a string with $(p, q)$ charge $(1,0))$ attached to the D3 brane at $\left(0, U_{\max }\right)$ and a $\mathrm{D}$-string $\left(\right.$ i.e. a $(0,1)$ string) attached to the $\mathrm{D} 3$ brane at $\left(L, U_{\max }\right)$. These two strings are attached to each other at the point $\left(\Delta L, U_{0}\right)$. However, in order that the $(p, q)$ charge is conserved, there must be another string with outgoing charge $(1,1)$ attached to the other strings at the point $\left(\Delta L, U_{0}\right)$. The other end of this string is attached to one of the D3 branes at $\left(\Delta L, U_{0}\right)$. This configuration is shown in Figure 1.

From the Yang-Mills perspective on the branes, a quark and a monopole can bind together to form a dyon, so when the distance separation is zero, we should be left with only with a $(1,1)$ string that stretches from $U=0$ 


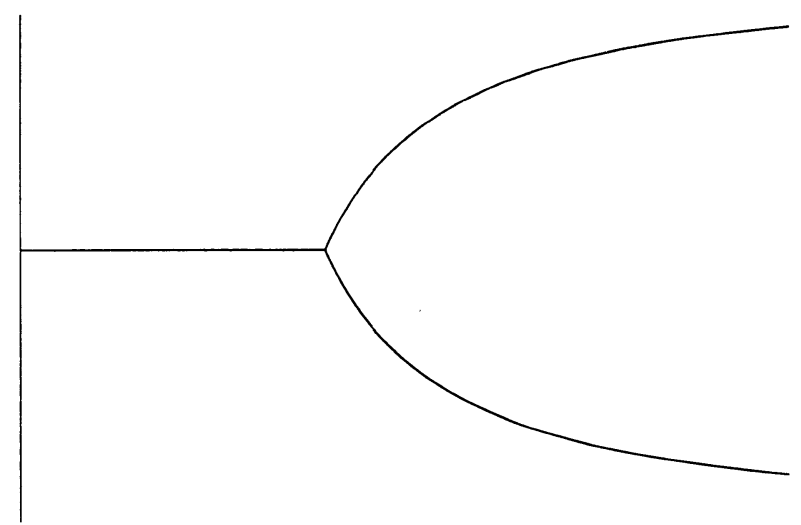

Figure 1: Three string-junction, with $(1,0)$ and $(0,1)$ strings coming in from $U=\infty$ and the $(-1,-1)$ string attached to the $N$ D3 branes at $U=0$.

to $U=\infty$. As the quark and the monopole move apart, the $(1,1)$ string remains attached to one of the branes but its length gets shorter. From the string picture, this sort of looks like we are pulling open a zipper.

We can now turn to the world-sheet action to find a minimum energy configuration. Since the dilaton field is constant for a background with parallel D3 branes, the world sheet action for any $(p, q)$ string in this background is given by (2.2), multiplied by a factor of $\sqrt{p^{2}+q^{2} t^{2}}$, where $t=1 / g$ and we are assuming that the theta angle is zero. Clearly, the $(1,1)$ string contributes $U_{0} \sqrt{1+t^{2}} /(2 \pi)$ to the total energy. As for the other two strings, we need to minimize the action in (2.3) for each string, giving us the equations

$$
\frac{U^{4}}{\sqrt{\left(\partial_{x} U\right)^{2}+U^{4} / R^{4}}}=U_{i}{ }^{2} / R^{2} \quad i=1,2,
$$

where $i=1(i=2)$ is the result for the fundamental string (D-string). Notice that $U_{i}$ is not necessarily equal to $U_{0}$. Proceeding as before, we find that the lengths of the two strings are

$$
\begin{aligned}
\Delta L & =\frac{\alpha_{1} R^{2}}{U_{0}} \int_{\alpha_{1}}^{\infty} \frac{d y}{y^{2} \sqrt{y^{4}-1}} \\
L-\Delta L & =\frac{\alpha_{2} R^{2}}{U_{0}} \int_{\alpha_{2}}^{\infty} \frac{d y}{y^{2} \sqrt{y^{4}-1}}
\end{aligned}
$$

where $\alpha_{i}=U_{0} / U_{i}$. Notice that $\alpha_{i} \geq 1$, otherwise the integral will not be real. If $\alpha_{i}>1$, then the tangent vector along the string will jump when going from the $(1,0)$ string to the $(0,1)$ string. 
We can now plug these expressions back into (2.2) and find the energy for this configuration. As in the quark-quark case, the energy diverges because of the quark and monopole masses. Subtracting off $U_{\max } /(2 \pi)$ for the quark mass and $t U_{\max } /(2 \pi)$ for the monopole mass, we are left with a finite energy, given by

$$
\begin{aligned}
E_{Q M}=\frac{U_{0}}{2 \pi}\left[-1+\frac{1}{\alpha_{1}} \int_{\alpha_{1}}^{\infty} d y\left(\frac{y^{2}}{\sqrt{y^{4}-1}}-1\right)-t\right. \\
\left.\qquad \frac{t}{\alpha_{2}} \int_{\alpha_{2}}^{\infty} d y\left(\frac{y^{2}}{\sqrt{y^{4}-1}}-1\right)+\sqrt{1+t^{2}}\right]
\end{aligned}
$$

We can use (3.2) to solve for $U_{0}$ in terms of $\alpha_{1}$ and $\alpha_{2}$. Substituting this into (3.3) we can rewrite $E_{Q M}$ as

$$
E_{Q M}=\frac{R^{2}}{2 \pi L}\left(F\left(\alpha_{1}\right)+F\left(\alpha_{2}\right)\right)\left(G\left(\alpha_{1}\right)+t G\left(\alpha_{2}\right)+\sqrt{1+t^{2}}\right),
$$

where the functions $F(\alpha)$ and $G(\alpha)$ can be written in terms of the Gaussian hypergeometric functions and are given by

$$
F(\alpha)=\frac{1}{3 \alpha^{2}} F\left(\frac{1}{2}, \frac{3}{4} ; \frac{7}{4} ; \frac{1}{\alpha^{4}}\right) \quad G(\alpha)=-F\left(\frac{1}{2},-\frac{1}{4} ; \frac{3}{4} ; \frac{1}{\alpha^{4}}\right)
$$

We now want to adjust $\alpha_{1}$ and $\alpha_{2}$ such that $E_{Q M}$ is minimized. We can take derivatives with respect to $\alpha_{1}$ and $\alpha_{2}$, set them to zero, and solve for $\alpha_{1}$ and $\alpha_{2}$. In the end this seems to involve proving an obscure identity for hypergeometric functions (see the appendix).

A much easier way to proceed is to adjust $\alpha_{1}$ and $\alpha_{2}$ such that the net force at the string junction is zero. If this were not zero, then the junction could move and lower the energy. For the $(1,0)$ and $(0,1)$ string, the derivatives $\partial_{x} U$ at $U=U_{0}$ are $\partial_{x} U=-U_{0}^{2} \sqrt{\left(U_{0} / U_{1}\right)^{4}-1} / R^{2}$ and $\partial_{x} U=U_{0}^{2} \sqrt{\left(U_{0} / U_{2}\right)^{4}-1} / R^{2}$. Moreover the infinitesmal lengths squared along the strings are $d s^{2}=\alpha^{\prime} U_{0}{ }^{6} /\left(U_{1}{ }^{4} R^{2}\right) d x^{2}$ and $d s^{2}=\alpha^{\prime} U_{0}{ }^{6} /\left(U_{2}{ }^{4} R^{2}\right) d x^{2}$. Hence, from (2.3) we see that the tensions of the strings at $U=U_{0}$ are

$$
T_{1,0}=\frac{1}{2 \pi} \frac{U_{0}^{4}}{U_{1}^{2} R^{2}} \frac{d x}{d s}=\frac{1}{2 \pi \sqrt{\alpha^{\prime}} R} U_{0} \quad T_{0,1}=\frac{1}{2 \pi \sqrt{\alpha^{\prime}} R} t U_{0}
$$

The tension of the $(1,1)$ string is $U_{0}\left(2 \pi \sqrt{\alpha^{\prime}} R\right)^{-1} \sqrt{1+t^{2}}$. Therefore, the forces exerted by each of the strings in the $x-U$ plane are

$$
\begin{aligned}
\vec{F}_{1,0} & =\left(-\left(U_{1} / U_{0}\right)^{2}, \sqrt{1-\left(U_{1} / U_{0}\right)^{4}}\right) \frac{U_{0}}{2 \pi \sqrt{\alpha^{\prime}} R} \\
\vec{F}_{0,1} & =\left(\left(U_{2} / U_{0}\right)^{2}, \sqrt{1-\left(U_{2} / U_{0}\right)^{4}}\right) \frac{U_{0} t}{2 \pi \sqrt{\alpha^{\prime}} R} \\
\vec{F}_{-1,-1} & =(0,-1) \frac{U_{0} \sqrt{1+t^{2}}}{2 \pi \sqrt{\alpha^{\prime}} R} .
\end{aligned}
$$


Clearly the net force is zero if $\alpha_{1}{ }^{4}=\left(U_{0} / U_{1}\right)^{4}=\left(1+t^{2}\right) / t^{2}$ and $\alpha_{2}{ }^{4}=$ $\left(U_{0} / U_{2}\right)^{4}=1+t^{2}$. Substituting these expressions back into (3.4), we find the expression

$$
\begin{aligned}
& E_{Q M}= \\
& -\frac{\sqrt{4 \pi N}}{6 \pi L \sqrt{g\left(1+g^{2}\right)}}\left(g F\left(\frac{1}{2},-\frac{1}{4} ; \frac{3}{4} ; \frac{1}{1+g^{2}}\right)+F\left(\frac{1}{2},-\frac{1}{4} ; \frac{3}{4} ; \frac{g^{2}}{1+g^{2}}\right)-\sqrt{1+g^{2}}\right) \\
& \quad \times\left(F\left(\frac{1}{2}, \frac{3}{4} ; \frac{7}{4} ; \frac{1}{1+g^{2}}\right)+g F\left(\frac{1}{2}, \frac{3}{4} ; \frac{7}{4} ; \frac{g^{2}}{1+g^{2}}\right)\right) .
\end{aligned}
$$

As in the quark-quark case, the potential falls off as $1 / L$, as is required by conformal invariance. Although perhaps not immediately obvious from the expression, $E_{Q M}$ is negative for all $g$ (A graph of $E_{Q M}$ versus $g$ is shown in Figure 2). Moreover, $E_{Q M}$ is manifestly invariant under the $S$ duality transformation $g \rightarrow 1 / g$, even though strictly speaking, the binding energy is not invariant. For large but finite $U_{\max }$, the binding energy is $B_{Q M}=$ $\left(\sqrt{1+1 / g^{2}}-1-1 / g\right) U_{\max } /(2 \pi)$, so under $g \rightarrow 1 / g, B_{Q M} \rightarrow g B_{Q M}$. Of course, in our calculation $B_{Q M} \rightarrow \infty$, so the $S$ duality invariance for the quark-monopole potential is valid in the region where $L>>\sqrt{g N}\left(U_{\max }\right)^{-1}$ and $L>>\sqrt{N / g}\left(U_{\max }\right)^{-1}$.

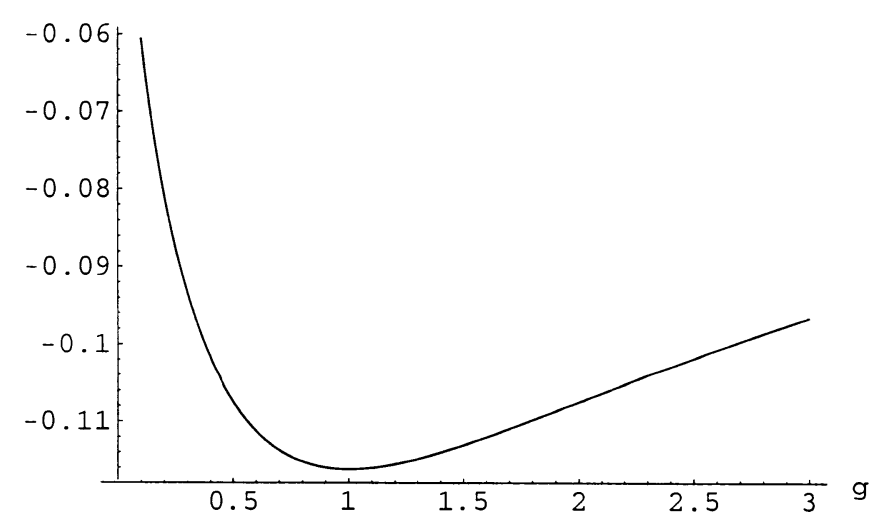

Figure 2: Plot of $E_{Q M} L / \sqrt{N}$ vs. $g . E_{Q M}$ is negative for all $g$.

Note that we can replace the $(0,1)$ and $(1,1)$ strings with the $(0,-1)$ and $(1,-1)$ strings. This corresponds to replacing the monopole with its charge conjugate. The calculation proceeds as before and we find the same attractive potential (at least when $\theta=0$ ).

It is instructive to compare the quark-monopole potential with the quarkquark potential at the self dual point. Letting $g=1$, one can easily evaluate the expressions in (2.7) and (3.8), and find that $E_{Q M} \approx .143 E_{Q \bar{Q}}$ at $g=1$. This is consistent with the reasoning presented above. 
There is also interesting behavior as $g$ becomes large or small (but still satisfying $4 \pi g N>>1$ and $4 \pi N / g>>1$ ). As $g \rightarrow \infty$, we have

$$
\begin{aligned}
F\left(\frac{1}{2}, \frac{3}{4} ; \frac{7}{4} ; \frac{g^{2}}{1+g^{2}}\right) & =\frac{3 \sqrt{2 \pi^{3}}}{(\Gamma(1 / 4))^{2}}+\mathrm{O}\left(g^{-2}\right) \\
F\left(\frac{1}{2},-\frac{1}{4} ; \frac{3}{4} ; \frac{1}{1+g^{2}}\right) & =1+\mathrm{O}\left(g^{-2}\right) \\
F\left(\frac{1}{2},-\frac{1}{4} ; \frac{3}{4} ; \frac{g^{2}}{1+g^{2}}\right) & =\frac{\sqrt{2 \pi^{3}}}{(\Gamma(1 / 4))^{2}}+\mathrm{O}\left(g^{-2}\right) .
\end{aligned}
$$

Hence, we find that potential is approximately

$$
E_{Q M}=-\frac{\pi^{2}\left(4 \pi g^{-1} N\right)^{1 / 2}}{L(\Gamma(1 / 4))^{4}}+\mathrm{O}\left(g^{-3 / 2}\right)=-\frac{4 \pi^{3}\left(g_{Y M}^{-2} N\right)^{1 / 2}}{L(\Gamma(1 / 4))^{4}}+\mathrm{O}\left(g_{Y M}{ }^{-3}\right)
$$

This is $1 / 4$ the potential for two monopoles. Likewise, in the limit that $g \rightarrow 0$, we find that the potential is $1 / 4$ the potential found for two quarks.

\section{Acknowledgements}

I would like to thank J. Maldacena and other participants of Dualities in String Theory '98 for valuable discussions. I would also like to thank the ITP at Santa Barbara for its hospitality during the course of this work. This work was supported in part by funds provided by the DOE under grant number DE-FG03-84ER-40168.

\section{Appendix: Minimizing the Energy}

Taking an $\alpha_{1}$ derivative on $E_{Q M}$ in (3.4) and (3.5), we find

$$
\begin{aligned}
& \frac{\partial E_{Q M}}{\partial \alpha_{1}}=-\frac{R^{2}}{6 \pi L} \frac{1}{\alpha_{1}^{3} \sqrt{\alpha_{1}^{4}-1}} \times \\
& \times\left[\left(\sqrt{t^{2}+1}-F\left(\frac{1}{2},-\frac{1}{4} ; \frac{3}{4} ; \frac{1}{\alpha_{1}^{4}}\right)-t F\left(\frac{1}{2},-\frac{1}{4} ; \frac{3}{4} ; \frac{1}{\alpha_{2}^{4}}\right)\right)\right. \\
& \times\left(3 \alpha_{1}^{2}-\sqrt{\alpha_{1}^{4}-1} F\left(\frac{1}{2}, \frac{3}{4} ; \frac{7}{4} ; \frac{1}{\alpha_{1}^{4}}\right)\right) \\
&+\left(1-\sqrt{\alpha_{1}^{4}-1} F\left(\frac{1}{2},-\frac{1}{4} ; \frac{3}{4} ; \frac{1}{\alpha_{1}^{4}}\right)\right) \\
& \\
&\left.\quad \times\left(F\left(\frac{1}{2}, \frac{3}{4} ; \frac{7}{4} ; \frac{1}{\alpha_{1}^{4}}\right)+\frac{\alpha_{1}^{2}}{\alpha_{2}^{2}} F\left(\frac{1}{2}, \frac{3}{4} ; \frac{7}{4} ; \frac{1}{\alpha_{2}{ }^{4}}\right)\right)\right] .
\end{aligned}
$$


If we now let $\alpha_{1}=\sqrt{\sqrt{1+t^{2}} / t}$ and $\alpha_{2}=\sqrt{\sqrt{1+t^{2}}}$, then (A.1) reduces to

$$
\begin{aligned}
& \frac{\partial E_{Q M}}{\partial \alpha_{1}}=-\frac{R^{2}}{6 \pi L}\left(\frac{t^{2}}{1+t^{2}}\right)^{3 / 4} \times \\
& \times\left[t F\left(\frac{1}{2}, \frac{3}{4} ; \frac{7}{4} ; \frac{t^{2}}{1+t^{2}}\right) F\left(\frac{1}{2},-\frac{1}{4} ; \frac{3}{4} ; \frac{1}{1+t^{2}}\right)\right. \\
&- t^{-1} F\left(\frac{1}{2}, \frac{3}{4} ; \frac{7}{4} ; \frac{1}{1+t^{2}}\right) F\left(\frac{1}{2},-\frac{1}{4} ; \frac{3}{4} ; \frac{t^{2}}{1+t^{2}}\right) \\
&+\frac{\sqrt{1+t^{2}}}{t} F\left(\frac{1}{2}, \frac{3}{4} ; \frac{7}{4} ; \frac{1}{1+t^{2}}\right)-\sqrt{1+t^{2}}\left(F\left(\frac{1}{2},-\frac{1}{4} ; \frac{3}{4} ; \frac{t^{2}}{1+t^{2}}\right)\right. \\
&\left.\left.+t F\left(\frac{1}{2},-\frac{1}{4} ; \frac{3}{4} ; \frac{1}{1+t^{2}}\right)\right)+3\left(1+t^{2}\right)\right]
\end{aligned}
$$

The term inside the square brackets is identically zero. A similar calculation can be done for $\frac{\partial E_{Q M}}{\partial \alpha_{1}}$.

\section{References}

[1] A. Polyakov, "String theory and quark confinement", hep-th/9711002.

[2] J. Maldacena, "The large $N$ limit of superconformal field theories and supergravity", hep-th/9711200.

[3] S. Ferrara and C. Fronsdal, "Conformal Maxwell theory as a singleton field theory on $\operatorname{AdS}(5)$, IIB three-branes and duality", hepth/9712239.

[4] P. Claus, R. Kallosh, J. Kumar, P. Townsend, and A. V. Proeyen, "Conformal theory of M2, D3, M5 and D1-branes + D5-branes", hepth/9801206.

[5] N. Itzhaki, J. Maldacena, J. Sonnenschein and S. Yankielowicz, "Supergravity and the large $N$ limit of theories with 16 supercharges", hep-th/9802042.

[6] S. Ferrara and C. Fronsdal, "Gauge fields as composite boundary excitations", hep-th/9802126.

[7] S. Ferrara, C. Fronsdal, and A. Zaffaroni, "On $N=8$ supergravity on $\operatorname{AdS}(5)$ and $N=4$ superconformal yang-mills theory", hepth/9802203.

[8] S. Gubser, I. Klebanov and A. Polyakov, "Gauge theory correlators from noncritical string theory", hep-th/9802109. 
[9] G. Horowitz and H. Ooguri, "Spectrum of large $N$ gauge theory from supergravity", hep-th/9802116.

[10] E. Witten, "Anti de Sitter space and holography", hep-th/9802150.

[11] S. Kachru and E. Silverstein, "4d conformal field theories and strings on orbifolds", hep-th/9802183.

[12] A. Lawrence, N. Nekrasov, and C. Vafa, "On conformal field theories in four-dimensions", hep-th/9803015.

[13] S.-J. Rey and J. Yee, "Macroscopic strings as heavy quarks in large $N$ gauge theory and anti-de Sitter supergravity", hep-th/9803001.

[14] J. M. Maldacena, "Wilson loops in large $N$ field theories", hepth/9803002.

[15] M. Berkooz, "A supergravity dual of a $(1,0)$ field theory in sixdimensions", hep-th/9802195.

[16] O. Aharony, Y. Oz and Z. Yin, "M Theory on $A d S_{p} \times S^{11-p}$ and Superconformal Field Theories, hep-th/9803051.

[17] S. Minwalla, "Particles on $A d S_{4 / 7}$ and Primary Operators on $M_{2 / 5}$ Brane Worldvolumes", hep-th/9803053.

[18] M. Flato and C. Fronsdal, "Interacting singletons", hep-th/9803013.

[19] S. Ferrara and A. Zaffaroni, " $N=1,24 D$ Superconformal Field Theories and Supergravity in $A d S_{5}$ ", hep-th/9803060.

[20] M.J. Duff, H. Lu and C.N. Pope, "Gauge theory $\rightarrow I I B \rightarrow I I A \rightarrow M$ duality", hep-th/9803061.

[21] R. Leigh and M. Rozali, "The Large $N$ Limit of the $(2,0)$ Superconformal Field Theory", hep-th/9803068.

[22] M. Bershadsky, Z. Kakushadze and C. Vafa, "String Expansion as Large N Expansion of Gauge Theories", hep-th/9803076.

[23] Edi Halyo, "Supergravity on $A d S_{4 / 7} \times S^{7 / 4}$ and $M$ Branes", hepth/9803077.

[24] G. Horowitz and S. Ross, "Possible Resolution of Black Hole Singularities from Large $N$ Gauge Theory", hep-th/9803085.

[25] O. Aharony, J. Sonnenschein and S. Yankielowicz, Nucl. Phys. B474 (1996) 309. 
[26] J. H. Schwarz, Nucl. Phys. Proc. Suppl. 55B (1997) 1.

[27] K. Dasgupta and S. Mukhi, "BPS Nature of 3-String Junctions", hep-th/9711094.

[28] A. Sen, "String Network", hep-th/9711130.

[29] S-J. Rey and J-T. Yee, "BPS Dynamics of Triple $(p, q)$ String Junction", hep-th/9711202.

[30] M. Krogh and S. Lee, "String Network from M-theory", hepth/9712050.

[31] Y. Matsuo and K. Okuyama, "BPS Condition of String Junction from $M$ theory", hep-th/9712070.

[32] O. Bergman, "Three-Pronged Strings and $1 / 4 B P S$ States in $N=4$ Super-Yang-Mills", hep-th/9712211.

[33] M. R. Gaberdiel, T. Hauer and B. Zwiebach, "Open string - string junction transitions", hep-th/9801205.

[34] C.G. Callan, L. Thorlacius, "Worldsheet Dynamics of String Junctions", hep-th/9803097. 